\title{
VLSI Implementation of Multiply and Accumulate Unit Using Distributed Arithmetic
}

\author{
Rajyalakshmi Chikkani', Bharathi. $\mathrm{M}^{2}$ and Yasha Jyothi M Shirur ${ }^{3}$ \\ ${ }^{1} M$. Tech Student, Sree Vidyanikethan Engineering College, Tirupati, Andhra Pradesh, India \\ ${ }^{2}$ Research Scholar, VTU, Assistant Professor, Department of ECE, Center \\ for VLSI \& Embedded Systems, Sree Vidyanikethan, India \\ ${ }^{3}$ Tirupati, Andhra Pradesh and Professor of ECE, BNMIT, Bangalore, VTU, India.
}

\section{ABSTRACT}

Background: DSP is used in many applications such as modems, audio broadcast, multimedia applications, cell phones, video broadcast, etc., DSP processors are concerned essentially with on-going signal processing. The "multiply and Accumulate Unit”, It is dual operand digital signal processing instructions. MAC is considered important in all DSP architectures. MAC comprises multiplier, adder, and accumulator. Speed, area and performance are the major constraints that have to be considered. Methods: On considering DA method which replaces the MAC with pre-computed results about to store in lookup tables. DA based on the memory-based method is trending engineering since of its high performance. The derivation of DA includes a mix of Boolean and ordinary algebra. DA is a bit-serial arrangement of multiply-accumulate. The most advantage of DA is consumed the low area, high speed, and low power.

Results: Implementation of actual DA with different architectures use in a traditional lookup table (LUT) based DAimplementation such as DA-based implementation use in Single LUT, DA based implementation use in double LUT, DA based implementation use in four LUTs, DA based implementation use in LUT and adder, adder based DAimplementation, 2BAAT DA structure with single LUT and double LUT and DA based implementation using zero detect logic.Finally, Comparing the overall performance in terms of area, power, and delay. Conclusions: Multiply and Accumulate (MAC) block design multiplier occupies more space than adder and accumulator since it consists of partial products, so area consumption of MAC block is more. So that delay increases, in this case, speed decreases. This is the main drawback of MAC. So, to overcome this drawback Distributed Arithmetic is preferred. Here an external multiplier block is not required and the multiplication is done through a look-up table. So partial product count will be reduced and delay decreases such that, area consumption also reduced and speed increases.

KEY WORDS: DISTRIBUTED ARITHMETIC, MULTIPLY-ACCUMULATE UNIT, LOOKUP TABLES(LUT), DIGITAL SIGNAL PROCESSOR, INNER PRODUCT COMPUTATION.

\section{ARTICLE INFORMATION}

Received 30th Oct 2020 Accepted after revision 13th Dec 2020 Print ISSN: 0974-6455 Online ISSN: 2321-4007 CODEN: BBRCBA

Thomson Reuters ISI Web of Science Clarivate Analytics USA and Crossref Indexed Journal

\section{Clarivate
Analytics}

NAAS Journal Score 2020 (4.31)

A Society of Science and Nature Publication,

Bhopal India 2020. All rights reserved.

Online Contents Available at: http//www.bbrc.in/

Doi: http://dx.doi.org/10.21786/bbrc/13.15/37

\section{INTRODUCTION}

Digital Signal Processing (DSP) is used in many applications such as modems, audio broadcast, multimedia applications, cell phones, video broadcast, and biomedical applications, etc., in ASIC architectures are used when the high-performance application is designed. This process continues because we are living in the world of System on a chip image and video processing applications are also 
implemented on a single chip. DSP has become a key part, in a large number of the shopper, interchanges, clinical and mechanical items which execute the sign preparing to utilize chip, Field Programmable Gate Arrays (FPGAs), Custom ICs and so on. DSP procedures have been exceptionally effective in light of the advancement of ease programming and equipment support. For instance, modems and discourse acknowledgment can be more affordable utilizing DSP systems. DSP processors are concerned essentially with ongoing signal processing.

In any DSP there will be a MAC unit. Multiply accumulate unit is also known as MAC. As the name itself, we can know that it is made up of a chain of multipliers, adders, and registers. First, inputs are passed to the multiplier and then the corresponding output will be obtained and that output will be sent to the adder and that output will be given to the accumulate register for data feedback. When speed is considered, to improve the speed of the MAC there are two ways.

1. Reduction of partial products and

2. Accumulator.

Figure 1: MAC Architecture

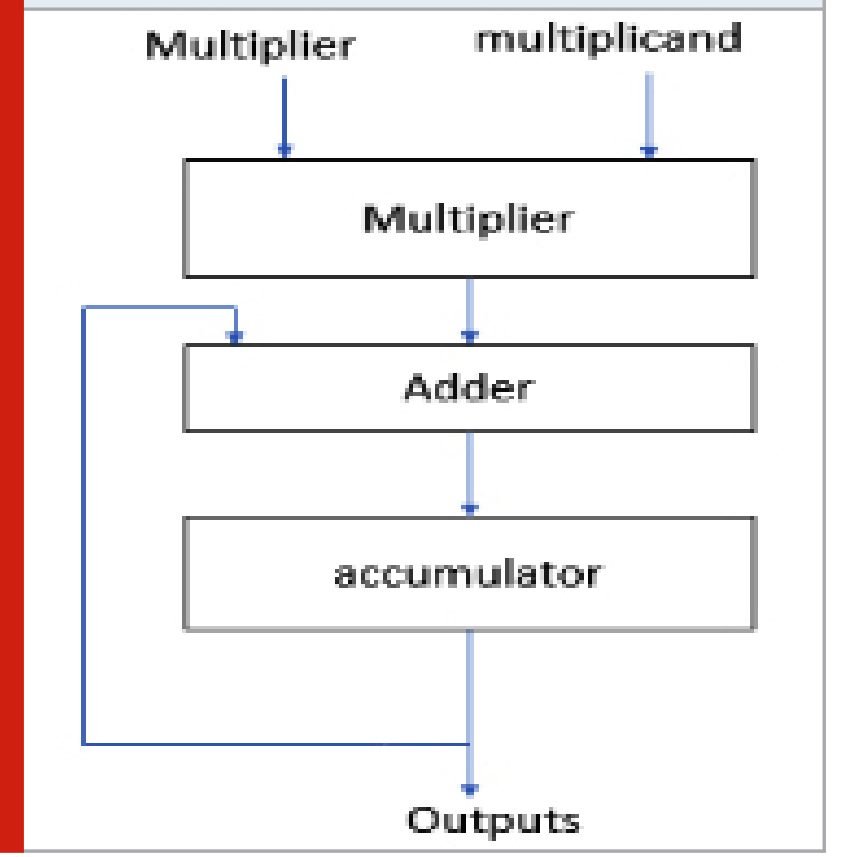

The above (figure1) describes the architecture of the general MAC, where multiplier and multiplicand are the two inputs to the multiplier, the operation of the multiplication is performed and that output is given to adder block as one of the input and second input is multiplier output that will be the other input to the adder. This is the way a complete MAC operation is performed. So many multipliers are used in MAC operations like Wallace, booth, modified booth, truncated and array multipliers. Implementation of multiply and accumulate unit is performed with all these types of the multiplier, adder and accumulator will be same for all MAC Units.. Among all these truncated MAC is not considered for general applications where accuracy is needed. It is considered when Accuracy is not preferred and like image and video processing applications, this type of multiplication is performed.

\section{METHODS}

As discussed, MAC contains a multiplier, adder, and accumulator. Since for this MAC unit is more area consumption and high delay. So, we are designing various MAC's based on different multipliers. Among them, the MAC designed with modified booth multiplier consumes less area, even though it consumes less area we can decrease that. So, to reduce the area consumption, Distributed Arithmetic is preferred. In Distributed Arithmetic multiplication is done without a multiplier. It uses LUT for the multiplication process. So, there will be no need for a partial product in this area consumption isdecreased.

Consider

$$
z=\sum_{a=1}^{a} i_{a} j_{a}
$$

is an N-bit two's complement number.

$$
\begin{aligned}
& \left|\mathrm{V}_{a}\right|<1 \\
& J_{a}:\left\{b_{a 0}, b_{k 1}, b_{a 2} \ldots \ldots \ldots, b_{a(n-1)}\right. \\
& \text { Where } b_{a 0} \text { is the sign bit } \\
& \text { - b. We can express } J_{a} \text { as } \\
& j_{a}=-b_{a 0}+\sum_{n=1}^{N-1} b_{a n} 2^{-n} \\
& z=-\sum_{a=1}^{a}\left(b_{a 0} * i_{a}\right)+\sum_{a=1}^{a}\left[\left(i_{a} * b_{a 1}\right) 2^{-1}+\left(i_{a} * b_{a 2}\right) 2^{-2}+\cdots+\left(i_{a}\right.\right. \\
& \left.\left.* b_{a(N-1)}\right) 2^{-(N-1)}\right] \\
& +\left[\left(b_{11} * i_{1}\right) 2^{-1}+\left(b_{12} * i_{1}\right) 2^{-2}+\cdots+\left(b_{1(N-1)} * i_{1}\right) 2^{-(N-1)}\right] \\
& z=-\left[b_{10} * i_{1}+b_{20} * i_{2}+b_{a 0} * i_{a}\right] \\
& +\left[\left(b_{11} * i_{1}\right) 2^{-1}+\left(b_{12} * i_{1}\right) 2^{-2}+\cdots+\left(b_{1(N-1)} * i_{1}\right) 2^{-(N-1)}\right] \\
& +\left[\left(b_{21} * i_{2}\right) 2^{-1}+\left(b_{22} * i_{2}\right) 2^{-2}+\cdots+\left(b_{2(N-1)} * i_{2}\right) 2^{-(N-1)}\right] \\
& +\left[\left(b_{a 1} * i_{a}\right) 2^{-1}+\left(b_{a 2} * i_{a}\right) 2^{-2}+\cdots+\left(b_{a(N-1)} * i_{a}\right) 2^{-(N-1)}\right] \\
& z=-\left[b_{10} * i_{1}+b_{20} * i_{2}+b_{a 0} * i_{a}\right] \\
& +\left[\left(b_{11} * i_{1}\right)+\left(b_{21} * i_{2}\right)+\cdots+\left(b_{a 1} * i_{a}\right)\right] 2^{-1}
\end{aligned}
$$

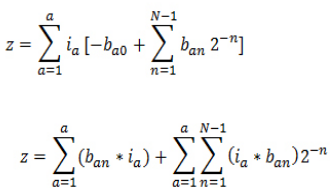


$+\left[\left(b_{12} * i_{1}\right)+\left(b_{22} * i_{2}\right)+\cdots+\left(b_{a 2} * i_{a}\right)\right] 2^{-1}$

$+\left[\left(b_{1(N-1)} * i_{1}\right)+\left(b_{2(N-1)} * i_{2}\right)+\cdots+\left(b_{a(N-1)} * i_{a}\right)\right] 2^{-(N-1)}$

$z=-\sum_{a=1}^{a}\left(b_{a 0}\right) * i_{a}+\sum_{n=1}^{N-1}\left[b_{1 n} * i_{a}+b_{2 n} * i_{2}+\cdots+b_{a n} * i_{a}\right] 2^{-n}$

$z=-\sum_{a=1}^{a} i_{a} *\left(b_{a 0}\right)+\sum_{n=1}^{N-1}\left[\sum_{a=1}^{a} i_{a} * b_{a n}\right] 2^{-n}$

Consider the equation (4) rewritten as:

$$
z=\sum_{n=1}^{N-1}\left[\sum_{a=1}^{a} i_{a} b_{\Xi}\right] 2^{-n}+\sum_{a=1}^{a} i_{a}\left(-b_{a 0}\right)
$$

- $\left[\sum_{a=1}^{a} i_{a} b_{a n}\right]$ has only $2^{\mathrm{a}}$ possible values

- $\left[\sum_{a=1}^{a} i_{a} b_{a n}\right]$ has only $2^{a}$ possible values

- With the sign bit as an input,we can store it in a ROM of size $=2^{*} 2^{a}$

Figure 2: DA-based implementation use in Single LUT

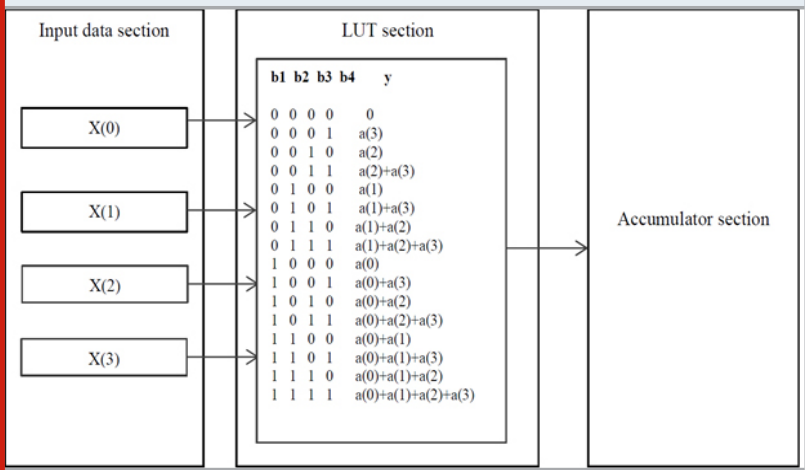

In the above expression, since each bnk sign bit takesonly 0 and 1 values, The bit values of 0 and 1 selected the input data section. In the input data section is given the LUT section. The operation of the LUT section is performed and that output is given the accumulator section. The output $\mathrm{Z}$ cycles are (A-1) additions and no multiplication. This is a way of complete DA based MAC operation.

Figure 3: DA based implementation use in double LUT



The above (figure 3)DA based implementation uses double LUT, Here anextra addition is performed in every cycle, For example k-term input data section, the number of address bits can be split into separate LUT. Each of the LUTs 2(k/2)rows, (figure 3) shows DA based implementation use in a single LUT, using two LUTs. The LUT size is 8 rows( 4 rows per LUT).

Figure 3: DA based implementation use in double LUT

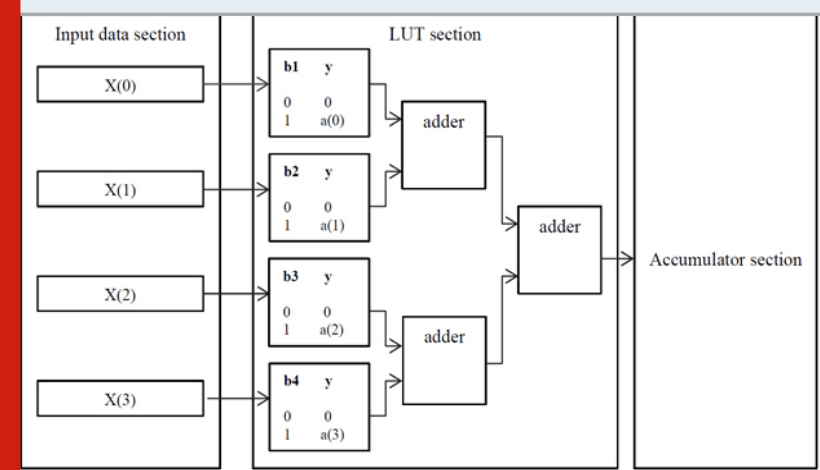

The above (figure 4)DA based implementation uses four LUTs, Here an extra addition is performed in every cycle. For example k-term input data section, the number of address bits can be split into separate LUT. Each of the LUTs 2(k/4)rows, (figure 4) shows DA based implementation use in a single LUT, using four LUTs. The LUT size is 8 rows( 2 rows per LUT).

Figure 4: DA based implementation use in four LUTs.

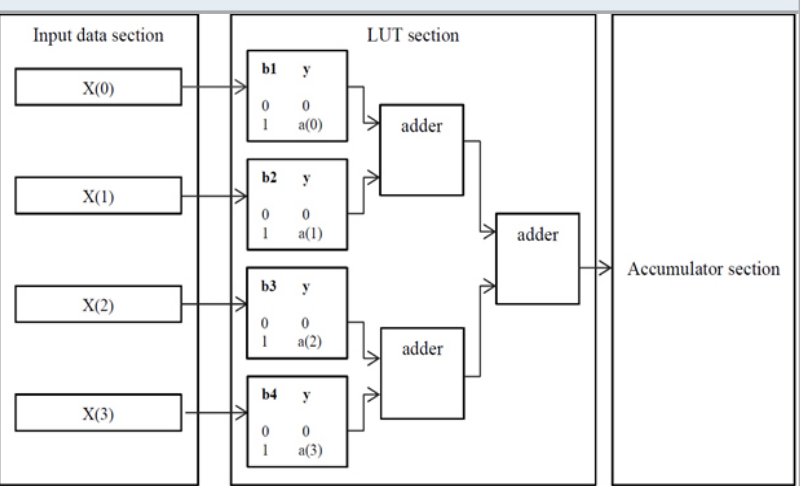

Figure 5: DA based implementation use in LUT and adder.

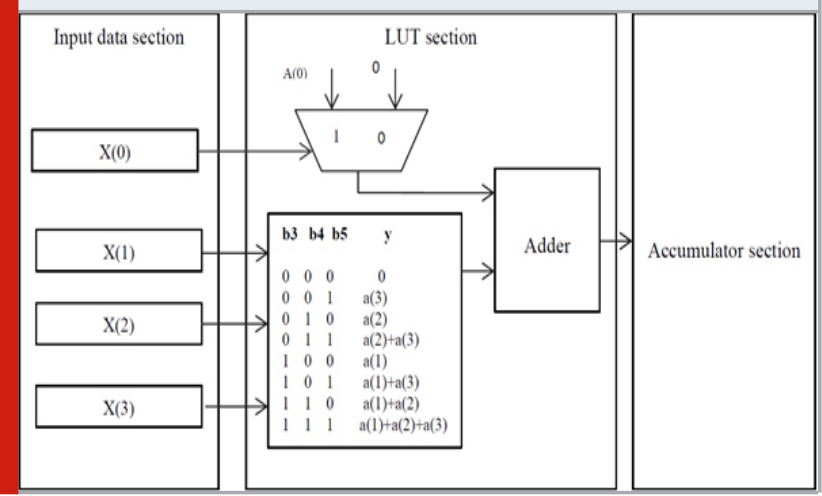

The above (figure 5) DA based implementation use in LUT and adder. It can be noted that the two contents one is the lower half of the LUT and the second one is 
the upper half by adding A[1]. The LUT size is reduced by half. It can be stored only the upper half of LUT and adding $\mathrm{A}[1]$ to LUT.

The above (figure 6) adder based DA implementation. It can be noted that the two contents one is the lower half of the LUT and the second one is the upper half by adding $\{\mathrm{A}[2]\}$. In this adder based DA implementation is applied tocompletely eliminate the LUT.

Figure 6: adder based DAimplementation.

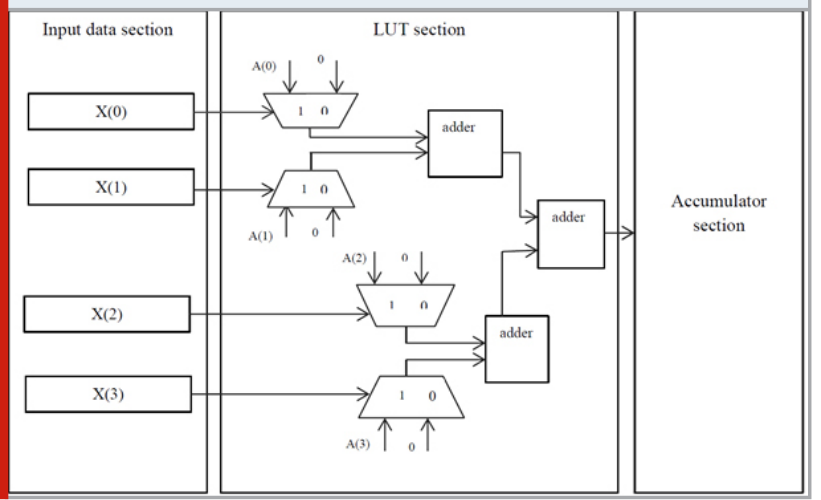

The above (figure 7) 2 BAAT DA architecture use in a single LUT. It is accessing the input data one bit at a time, which requires $\mathrm{N}$ cycles, It is accessed in two bits at aneach cycle, If two bits of input data are accessed in each clock cycle (2 BAAT), the number of address bits for the LUT gets doubled. For a structure of single LUT, this results in an increase the size of LUT by $2 \mathrm{~N}$ factor, the resultant architecture is shown in (figure 7).

Figure 7: 2 BAAT DA architecture used in a single LUT.

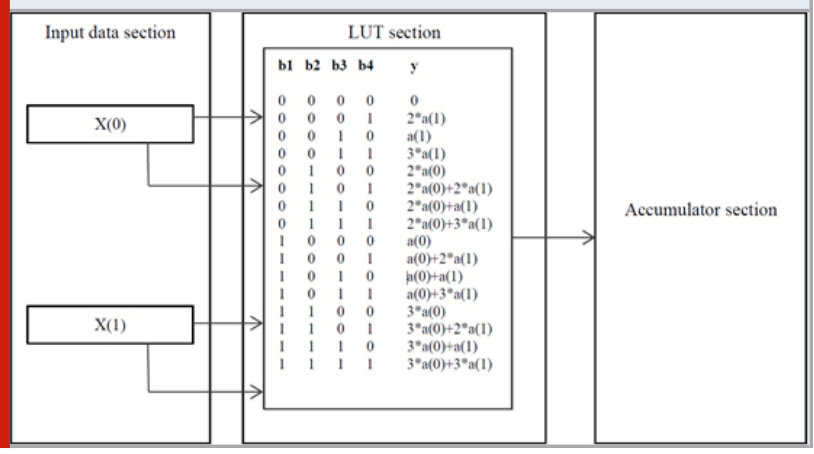

The above (figure 8) 2 BAAT DA architecture is used in double LUTs. Alternatively, the two LUTs are different weights. The size of LUT will be increased in two different factors. The below (figure 9) DA based implementation using zero detect logic.Since the entry of LUT is address "0" (all input bits 0) is 0, a zero detect logic on the LUT address bus can be used to disable LUT (figure 9). The amount of power reduction is dependent on the number of times the input bits at a given location are all zero.
Figure 8: 2 BAAT DA architecture used in double LUTs.

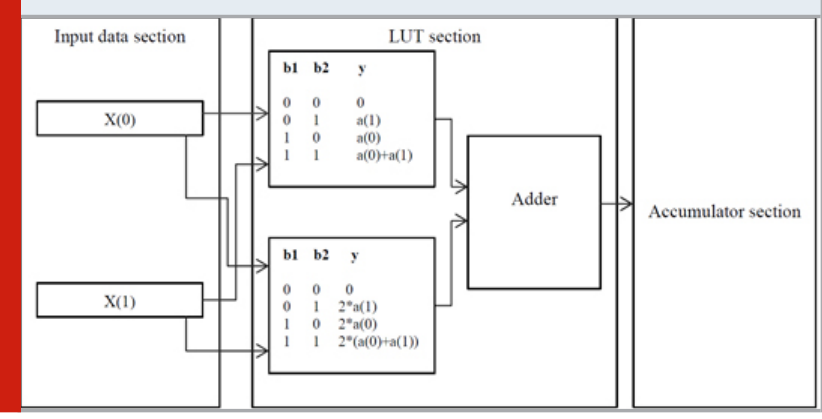

Figure 9: DA based implementation using zero detect logic.

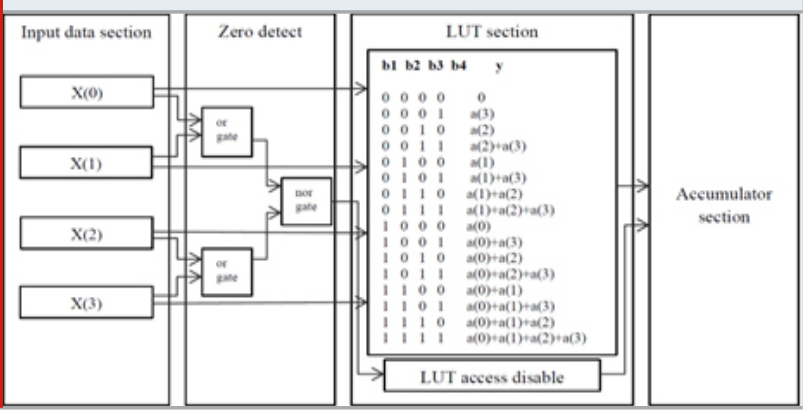

\section{RESULTS}

In this proposed work DA based implementation structures for MAC architectures has been designed using Verilog coding and simulation has been done using Xilinx ISE 14.7.
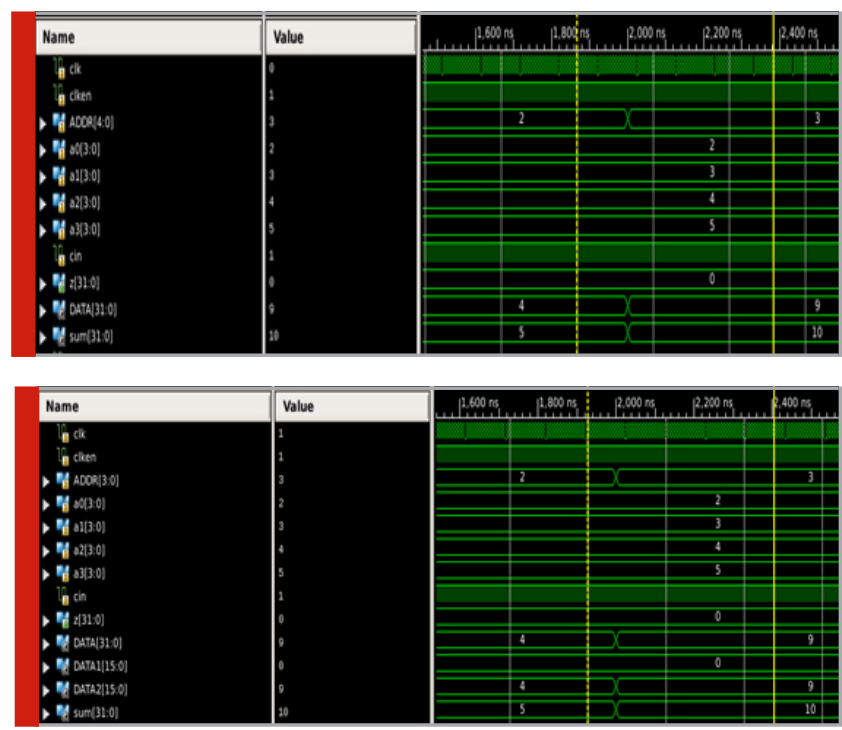

Simulation 1: DA-based implementation use in Single LUT.

a0,a1,a2,a3 = inputs $=2,3,4,5$

$\mathrm{ADDR}=0011=\mathrm{a} 2+\mathrm{a} 3=4+5=9=\mathrm{DATA}$

Sum $=$ DATA $+\operatorname{cin}=9+1=10$

$\mathrm{Clk}$, clken $=1$ then $\mathrm{z}=0$ else accumulation can be done 
Simulation 2: DA based implementation use in double LUT.

a0,a1,a2,a3 = inputs $=2,3,4,5$

$\mathrm{ADDR}=0011$

DATA $1=\mathrm{a} 0+\mathrm{a} 1=0$

DATA2 $=\mathrm{a} 3+\mathrm{a} 2=9$

DATA $=$ DATA $1+$ DATA2 $=0+9=9$

Sum $=$ DATA $+\operatorname{cin}=9+1=10$

$\mathrm{Clk}$, clken $=1$ then $\mathrm{z}=0$ else accumulation can be done

Performance evaluation of various DA based MAC in terms of Area:

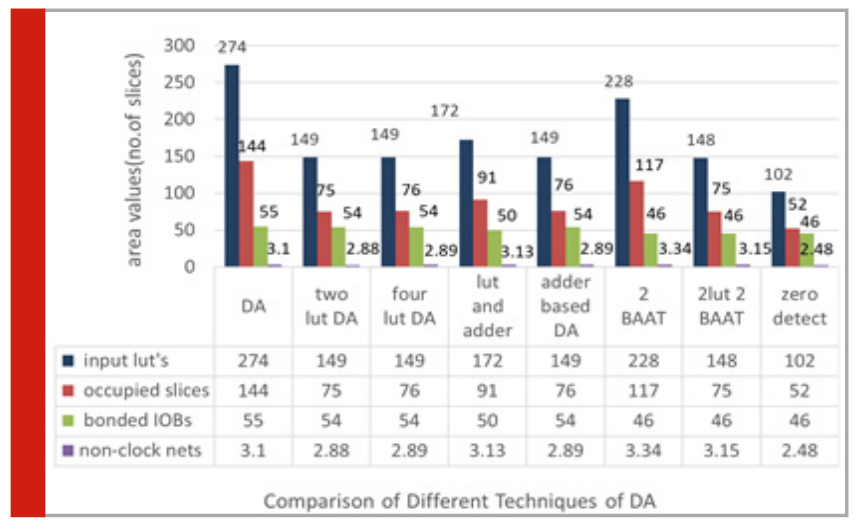

Here in DA architectures, the comparison is done with all types of DA architectures and compared. We have to give some of theDA based implementations using zero detect logic consumes less area among all types of DA architectures. From the above chart, DA consumes an area of 274 slices out of 17344 slices and DA based implementation using zero detect logicconsumes an area of 102 slices, so that area consumption is decreased.

\section{Performance evaluation of various DA based MAC in} terms of delay and energy:

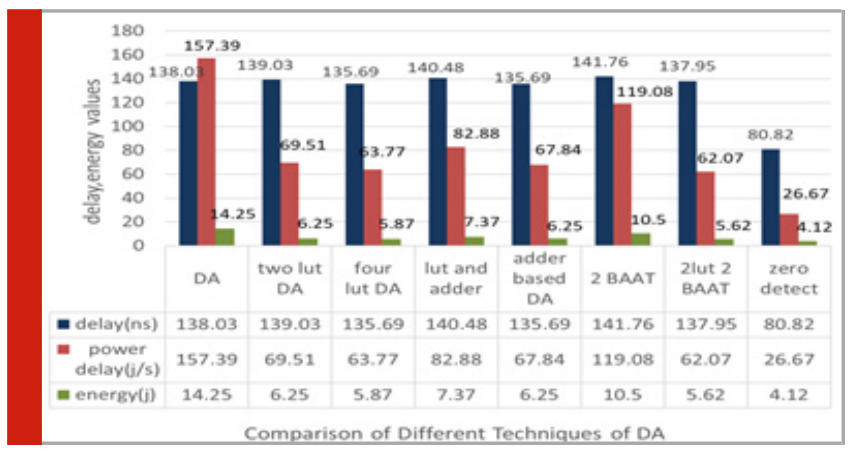

From the above chart, DA consumes a delay of 138.03ns DA based implementation using zero detect logicconsumes delay of $80.82 n s$, so that delay consumption is decreased.

Performance evaluation of various DA based MAC in terms of Power:

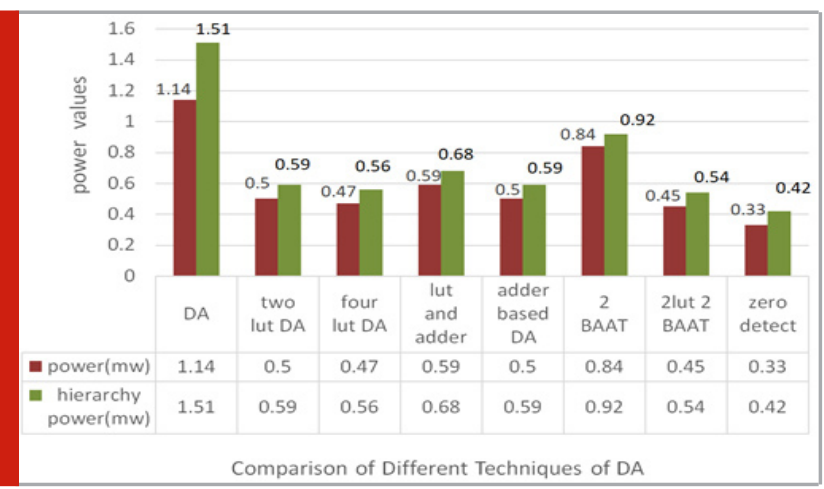

From the above chart, DA consumed a power of 1.14 mw and DA based implementation using zero detect logicconsumes a power of $0.33 \mathrm{mw}$, so that power consumption is decreased.

\section{CONCLUSION}

Multiply and Accumulate unit(MAC) block plays an important role in each and every digital signal processing(DSP), which consists of multiplier, adder and accumulator. In MAC block design, the multiplier occupies more space as an adder and accumulator since it consists of partial products, so area consumption of MAC block is more. So that delay increases, in this case speed decreases. This is the main drawback of MAC. So, to overcome this drawback, Distributed Arithmetic is preferred. Here, external multiplier block is not required and the multiplication is done through the look-up table. So the partial product count will be reduced and delay decreases such as that, area consumption also reduced and speed increases. So, area consumption increases a little. To overcome this drawback, Offset Binary Coding is selected. Here the ROM size is reduced to half, so that area consumption is completely reduced to half when compared with MAC. To increase a little more speed, the implementation of offset binary coding is done by replacing a normal adder with a floating-point adder. On considering, DA based implementation using zero detect logicarea consumption is decreased to $33 \%$ and delay is decreased to $55 \%$, but in this distributed arithmetic for each added input line ROM size grows exponentially. So, in offset binary coding, still, performance and area can also be improved. Designing Offset Binary Coding with Floating-point Adder still speed can be increased when compared with normal adder based on offset binary coding.

\section{ACKNOWLEDGEMENTS}

I might want to communicate my extraordinary gratefulness to Bharathi.M and Dr. Yasha Jyothi M Shirur for her significant and valuable proposals during the arranging and improvement of this research work.

\section{REFERENCES}

A.S. Remya Ajai and Nithin Nagarj, A Novel Methodology for Memory Reduction in Distributed Arithmetic Based Discrete Wavelet Transform, 2012, 
pp: 226-233.

Amita Nandal, T.Vigeswarn, Ashwani K. Rana and Arvind Dhaka, An Efficient 256-Tap Parallel FIR Digital Filter Implementation Using Distributed Arithmetic Architecture, 2015, pp: 605-611.

Basant Kumar Mohanty, Pramod Kumar Meher, Subodh Kumar singhal and M.N.S.Swamy, A highperformance VLSI architecture for reconfigurable FIR using distributed arithmetic, 2016, pp: 37-46.

D. Kalaiyarasi and T.Kalpalatha Reddy, Design and implementation of Least Mean Square adaptive FIR filter using offset binary coding based Distributed Arithmetic, November 2019.

Jiafeng Xie, jianjun He and guanzheng Tan, FPGA realization of FIR filters for high speed and medium speed by using modified distributed arithmetic architectures, June 2010, pp: 365-370.
M.Suhasini, K.Prabhu Kumar and P.Srinivas, Multiplier Design and Performance Estimation with Distributed Arithmetic Algorithm, 2012.

Mahesh Mehandale, Mohit Sharma, and Pramod Kumar Meher, DA-Based Circuits for Inner-Product Computation, pp:77-103.

Pranav J. Mankar, Ajinkya M. Pund, Kunl P.Ambhore and Shubham C. Anjankar, Design and Verification of low power DA-Adaptive digital FIR filter, 2016, pp: 367-373.

Priyanka Nain and Dr.G.S.Virdi, Mulitiplier Accumulator Unit, October 2016.

Rajeevan Amirtharajah, Distributed Arithmatic, pp-503512

Ramesh.R, Nathiya.R, Realization of FIR filter using Modified Distributed Arithmetic Architecture, 2012. Wayne P.Burleson, Louis L.Scharf, A VLSI design methodology for distributed arithmetic, June 1991 